\title{
Necrotizing Fasciitis of the Upper and Lower Limbs: Port Harcourt Experience
}

\author{
Rollings Jamabo, Solomon Elenwo \\ Department of Surgery, University of Port Harcourt Teaching Hospital, Port Harcourt, Nigeria. \\ Email: drjamabo@seasidesurgery.com
}

Received November 28 ${ }^{\text {th }}$, 2010; revised March 15 ${ }^{\text {th }}, 2011$; accepted April 6 ${ }^{\text {th }}, 2011$.

\begin{abstract}
Background: Necrotizing fasciitis (NF) is a rare but life-threatening soft-tissue infection characterized by rapidly spreading inflammation and subsequent necrosis of the fascial planes and surrounding tissue. Aim: To determine the clinical characteristics at presentation, causative pathogens and clinical outcome of NF after aggressive management. Patients and Methods: We retrospectively reviewed case notes of patients with NF referred to the Teaching Hospital in Port Harcourt from January 2004 to December 2009. Results: The case notes of over 2,280 patients with history of cellulitis and/or infections of the upper and lower limbs were reviewed. These cases were seen in a five-year period from 2004 to 2009. Seventy five (3.29\%) patients had a diagnosis of necrotizing fasciitis and were evaluated. Only those with infections on the lower and upper limbs were further analyzed. Thirty five (46.67\%) patients had their infections in the lower limb while 40 (53.33\%) patients had their infections in the upper limb. Twenty (26.67\%) patients were found to be diabetic on admission. Thirty (30\%) patients sustained injuries on their limbs while 5 (6.67\%) patients could not give account of their infections. Eleven (14.66\%) patients had fixed flexion deformities as their wounds healed and had to have further plastic surgery to extend their limbs and 7 (9.33\%) patients died while on admission. Conclusion: Although an early diagnosis of NF can be difficult, a high index of suspicion is required in all patients presenting with unexplained warmth and/or cellulitis of the limbs, so that prompt and aggressive debridement can be carried out with commencement of broad spectrum antibiotics.
\end{abstract}

Keywords: Necrotizing fasciitis, Causative Organisms, Treatment Outcome

\section{Introduction}

Necrotizing fasciitis (NF) is infrequently encountered in routine surgical practice. It is a rapidly progressive infection that affects the fascia and subcutaneous tissue concomitantly with the development of thrombosis of skin microcirculation, resulting in necrosis of skin and soft tissue [1]. The skin remains intact in the early stages of NF portraying a deceptive benign appearance but it is associated a severe localized pain, which is out of proportion to the size of the lesion. It is a poly-bacterial infection, associated with profound systemic toxicity, considerable morbidity and a high mortality rate. This disease on healing, especially those involving the limbs, leaves the patients with fixed flexion contractures of the joints.

We present 75 cases of NF of the upper and lower limbs that were seen in different stages of the disease in the hospital.

\section{Patients and Methods}

Between January 2004 and December 2009, there were 2,280 cases of cellulitis and/or infections of the upper and lower limbs admitted into the hospital. Relevant data as to the age, sex, history at presentation, clinical findings, and investigations especially those of microscopy, culture and sensitivity, antimicrobials used and the treatment outcome were obtained. The radiographs and photographs taken on admission were useful especially in those cases in which the disease had 'burnt out'.

\section{Results}

Of the 2,280 case notes of patients, only 75 (3.3\%) patients had proven diagnosis of necrotizing fasciitis of their lower and upper limbs. There were 41 (54.7\%) males and $34(45.3 \%)$ females. The ages ranged from 7 years to 60 years with a mean age of 41.1 years. Thirty-five (46.7\%) patients had their infection in the lower limb while 40 
(53.3\%) patients had theirs in the upper limb. Fifty (66.7\%) patients had identifiable premorbid pathology, of which $20(26.7 \%)$ were found to be diabetic on admission while $30(40 \%)$ sustained injuries on their limbs. Five (6.67\%) patients could not give account of their pre-morbid state. Seven (9.3\%) patients died while on admission. The most common clinical manifestation in all the cases include fever, pain in the limbs, swelling, redness, foul smelling discharge and skin necrosis. All the patients had extensive aggressive and extensive wound debridement and $11(14.66 \%)$ healed with fixed joint contractures (Figures 1-3). These contractures were released, with the raw areas grafted with a split skin graft and finally the limbs were held in extension with plaster of Paris until the wounds healed.

A total of approximately 100 wound cultures were carried out on these patients. Out of these, $67 \%$ were positive with growth of micro-organisms while $37 \%$ were negative. The commonest organisms grown were staphylococcus aureus, streptococci, bacteroides, pseudomonas aeruginosa and E.coli. All the patients were placed on cephalosparins, aminoglycosides and metronidazole.

\section{Discussion}

NF is a progressive, polymicrobial, potentially fatal soft tissue infection that can affect both sexes, all age groups and any anatomical region of the body [2]. It is an uncommon soft tissue infection, usually caused by toxinproducing virulent bacteria, which is characterized by widespread fascial necrosis with relative sparing of skin and underlying muscle [3]. In the early stages, the skin

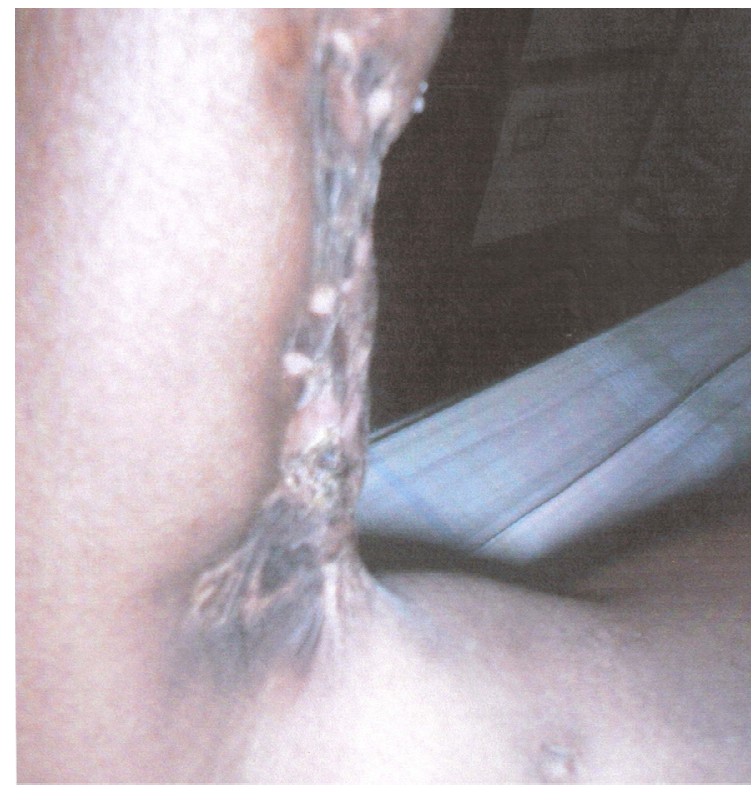

Figure 1. Fixed flexion deformity of the knee joint in one of the patients.

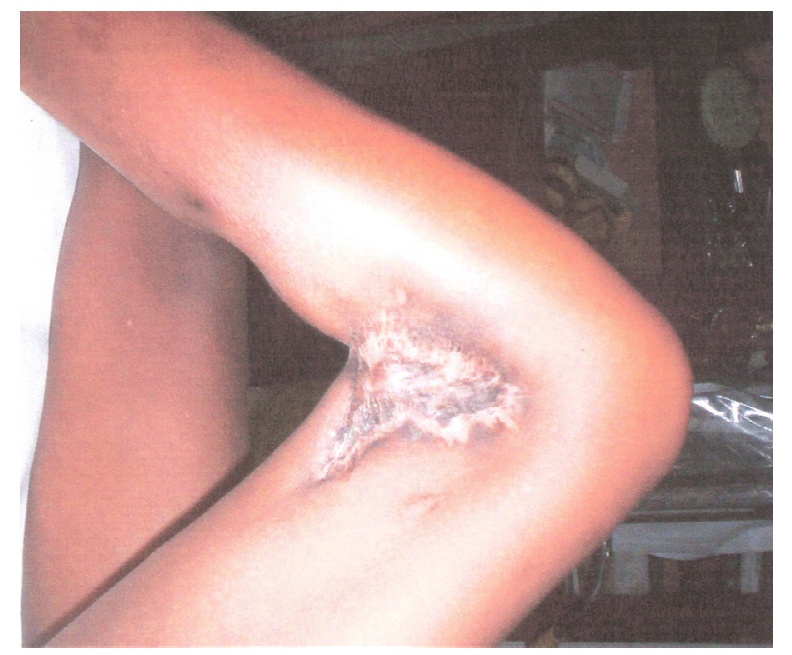

Figure 2. Fixed flexion deformity of the knee of one of the patients.

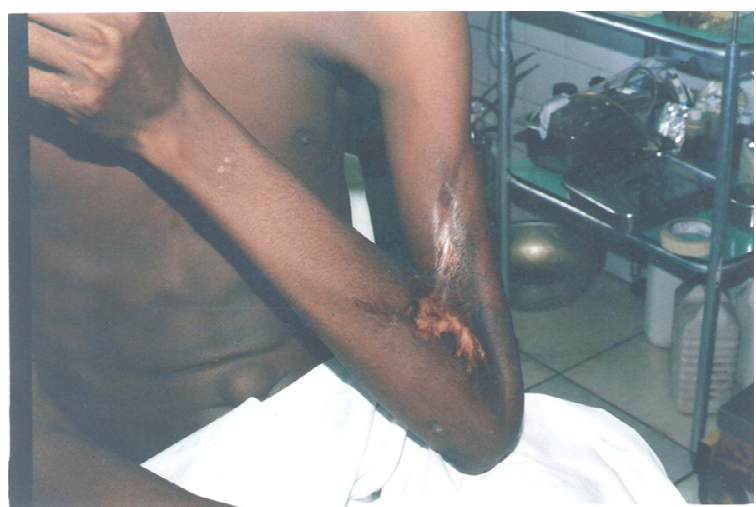

Figure 3. Fixed flexion deformity of the elbow of one of the patients.

remains intact and gives a deceptive benign appearance. As found in all our cases, the skin typically appears redhot, moderately swollen and exquisitely tender. Identification of the offending microorganisms is important, since the eventual outcome of treatment is dependent on aggressive surgical, chemotherapeutic and supportive therapy.

Three distinct NF syndromes are recognized [4]. These are the type 1 , which is the polymicrobial variety, type 2 , which is the monomicrobial variety of group A beta haemolytic streptococcus and type 3, which is the gas gangrene or clostridial myonecrosis variety. The type 2 or monomicrobial variety is less common and affects healthy individuals with history of trauma [4]. In our series, 70 patients were particularly unwell with various premorbid conditions and they were of the poly-microbial variety. The remaining 5 patients were healthy until they sustained minor trauma to their limbs.

Patients with NF often present with symptoms and 
signs of sepsis, systemic toxicity and evidence of skin inflammation, with pain that is disproportional to the degree of inflammation [5]. This severe local pain, which is out of proportion to the size and type of wound, is a hallmark sign seen in NF [6]. Because the condition is rare with minimal specific signs, early diagnosis can be difficult and requires a high index of suspicion in all patients presenting with cellulitis or unexplained sepsis [7]. Although diabetes mellitus is the most known commonest predisposing factor [8], only $26.7 \%$ of our cases were found to have diabetes mellitus. As farmers and fishermen, they sustained injuries on their lower limbs at various times and these were then secondarily infected by various organisms. This group formed $40 \%$ of the cases.

In most of our cases, there was a delay in making the correct diagnosis and commencing appropriate treatment for NF and this clearly increased the mortality. Clinical staging of the disease is being proposed by some workers [9] to better define the progression of the disease. Also several clinical subtypes such as hyperacute, acute and subacute variants of the disease have been described [9]. None of these have been universally accepted.

Wound swabs with microscopy, culture and sensitivity play a major role in the diagnosis and management of NF. In our study, over 100 microscopies, cultures and sensitivities were carried out with only 67 positive results. However, imaging techniques such as magnetic resonance imaging and frozen section biopsies which are reported to be of value in the early recognition of NF [10] were not available in our centre at the time of the study. Also the use of the fasciitis LRINEC (Laboratory Risk Indicator for Necrotizing fasciitis) score and transcutaneous tissue oxygen saturation monitoring, which have the potential for widespread application in the assessment of severe soft tissue infections [10] were not yet available in our centre at the time our study.

All our patients had aggressive debridement of their wounds and broad spectrum antibiotics which included cephalosporins, occasionally piperacillin with lactobatam, aminoglycosides and metronidazole.

\section{Conclusions}

Necrotizing fasciitis is a disease which has multiple aetiology and predisposing factors. A high index of suspicion is required in all patients presenting with unexplained warmth and/or cellulitis of the limbs. Early pres- entation and diagnosis prompt and aggressive debridement, commencement of broad spectrum antibiotics and supportive measures remains the cornerstone of management.

\section{Acknowledgements}

We wish to thank Professor Ndu Eke for reading through and making necessary corrections of the manuscript.

\section{REFERENCES}

[1] D. Huljev and N. Kucisec-Tepes, "Necrotizing Fasciitis of the Abdominal Wall as a Post-Surgical Complication: A Case Report," Wounds, Vol. 17, No. 7, 2005, pp. 169-177.

[2] J. N. Legbo and J. F. Legbo, "Bacterial Isolates From Necrotizing Fasciitis: A Clinico-Pathological Perspective," Nigerian Journal of Medicine, Vol. 16, No. 2, 2007, pp. 143-147.

[3] R. J. Green, D. C. Dafoe and T. A. Raffin, "Necrotizing Fasciitis,” Chest, Vol. 110, No. 1, 1996, pp. 219-229. doi:10.1378/chest.110.1.219

[4] R. Puvanendran, J. C. Huey and S. Pasupathy, "Necrotizing Fasciitis," Canadian Family Physician, Vol. 55, No. 10, 2009, pp. 981-987.

[5] E. J. Whallett, J. H. Stevenson and A. D. Wilmshurst, "Necrotizing Fasciitis of the Extremity," Journal of Plastic, Reconstructive \& Aesthetic Surger, Vol. 63, No. 5, 2010, pp. 469-473. doi:10.1016/j.bjps.2009.09.011

[6] S. D. Fritzsche, "Soft Tissue Infection: Necrotizing Fasciitis,” Plastic Surgery Nursing, Vol. 23, No. 4, 2003, pp. 155-159.

[7] M. Nisbet, G. Ansell, S. Lang, S. Taylor, P. Dzendrowskyj and D. Holland, "Necrotizing Fasciitis: Review of 82 Cases in South Auckland," Journal of Clinical Endocrinology \& Metabolism, Vol. 95, No. 1, 2010, pp. 11-12.

[8] A. Rajput, Waseem, A. Samad, T. W. Khanazada, G. M. Shaikh and G. A. Channa, "Mortality in Necrotizing Fasciitis,” Journal of Ayub Medical College Abbottabad, Vol. 20, No. 2, 2008, pp. 96-98.

[9] C. H. Wong and Y. S. Wang, "The Diagnosis of Necrotizing Fasciitis," Current Opinion in Infectious Diseases, Vol. 18, No. 2, 2005, pp. 101-106. doi:10.1097/01.qco.0000160896.74492.ea

[10] E. J. McGee, "Necrotizing Fasciitis: Review of Pathophysiology, Diagnosis and Treatment," Critical Care Nursing Quarterly, Vol. 28, No. 1, 2005, pp. 80-84. 